\title{
Téoros
}

Revue de recherche en tourisme

\section{Le CIFORT, un outil de l'internationalisation de l'UQAM}

\section{François Bédard et Boualem Kadri}

Volume 22, numéro 3, automne 2003

URI : https://id.erudit.org/iderudit/1071351ar

DOI : https://doi.org/10.7202/1071351ar

Aller au sommaire du numéro

Éditeur(s)

Université du Québec à Montréal

ISSN

0712-8657 (imprimé)

1923-2705 (numérique)

Découvrir la revue

Citer cette note

Bédard, F. \& Kadri, B. (2003). Le CIFORT, un outil de l'internationalisation de l'UQAM. Téoros, 22(3), 60-62. https://doi.org/10.7202/1071351ar d'utilisation que vous pouvez consulter en ligne.

https://apropos.erudit.org/fr/usagers/politique-dutilisation/ 
Centre international de formation et de recherche en tourisme

\section{Le CIFORT, un outil de I'internationalisation de I'UQAM}

\section{François Bédard et Boualem Kadri}

\begin{abstract}
$\Delta$ l'échelle mondiale, le tourisme se classe parmi les principaux secteurs en ce qui a trait à la création d'emplois et aux retombées économiques. En effet, près d'un emploi sur dix dans le monde est lié au secteur du tourisme et des voyages. Néanmoins, selon l'Organisation mondiale du tourisme (OMT), la progression accélérée de la mondialisation des produits, des services et des marchés ainsi que l'évolution croissante concernant l'utilisation des nouvelles technologies influent sur les programmes de formation et de recherche en tourisme.
\end{abstract}

Pour l'institution de formation et de recherche, en l'occurrence l'université, une telle situation véhicule de nouveaux enjeux et défis et induit à son encontre une double contrainte : d'une part, observer les effets enregistrés par l'industrie touristique (environnement de plus en plus concurrentiel, évolution des technologies, changement des comportements des consommateurs) et, d'autre part, surveiller le «marché » de la formation en mutation rapide (mise en œuvre de programmes d'e-formation par d'autres institutions, alliances stratégiques relatives au marché de la formation à distance entre diverses universités dans le monde).

Si les activités de formation et de recherche en gestion du tourisme ont toujours fait partie des caractéristiques distinctives de l'Université du Québec à Montréal (UQAM) depuis sa fondation, il importe pour cette université de demeurer proactive dans ses activités de formation et de recherche en tourisme et de renforcer sa présence et son rayonnement sur le plan international. Le CIFORT (Centre international de formation et de recherche en tourisme), s'inscrit alors dans ce processus d'internationalisation.

\section{Le CIFORT, un outil de concertation pour le développement de la formation et de la recherche à l'international}

Né en 1991 d'une initiative d'un groupe de professeurs du Département d'études urbaines et touristiques pour représenter l'UQAM à l'OMT, le CIFORT apparaît comme l'un des premiers centres à caractère international de cette université. La reconnaissance institutionnelle du Centre en juin 2003 vient consacrer ce dernier dans cette vocation qui a d'ailleurs permis de renforcer le processus d'internationalisation de l'UQAM.

Pour assurer le rayonnement international de l'université, le CIFORT s'est fixé une mission et des objectifs stratégiques, un mode d'organisation et une philosophie d'intervention. On peut en effet observer que la mission du CIFORT est structurée autour d'objectifs, tels que:

- développer la concertation entre les diverses instances de l'École des sciences de la gestion (ESG) de l'UQAM engagées dans la formation et la recherche en tourisme sur le plan international ;

- assurer la coordination des actions et des activités des diverses instances de l'ESG engagées en tourisme ;

- appuyer les diverses actions de l'ESG et de ses composantes liées au tourisme sur le plan international ;

- réaliser des mandats de recherche et de formation à l'échelle internationale ;

- représenter l'UQAM auprès de l'OMT dont elle est l'un des membres affiliés.

Rattaché depuis 2003 à l'École des sciences de la gestion de l'UQAM, tout en demeurant hébergé dans les locaux du département d'études urbaines et touristiques, le CIFORT s'est doté d'un comité de coordination composé de représentants provenant de diverses instances de l'ESG, dont :

- les programmes de premier cycle en gestion du tourisme et de l'hôtellerie,

- les programmes de deuxième cycle en gestion et planification du tourisme,

- le Département d'études urbaines et touristiques,

- la Chaire de tourisme,

- le Vice-décanat à la recherche de l'ESG,

- la revue Téoros,

- le Bureau de coopération internationale (BCI).

D'autres instances et personnes (professeurs, chargés de cours) viennent enrichir le CIFORT par leur contribution et partager diverses activités, notamment celles qui touchent la réalisation 
de mandats de formation et de recherche et ce, dans le cadre de la coopération internationale.

Le CIFORT et ses membres interviennent en offrant des services qui visent la formation et le développement des ressources humaines des secteurs de l'hôtellerie, de la restauration et du tourisme, notamment dans les domaines de la gestion, de la pédagogie et de la recherche. Par ailleurs, l'action du CIFORT est sous-tendue et animée par l'intérêt de faciliter l'appropriation par les partenaires immédiats de tous les résultats induits par l'intervention du CIFORT (réflexion, méthodologie, techniques, applications).

\section{Le CIFORT, un outil de l'internationalisation de I'UQAM}

L'engagement du CIFORT à l'international s'apprécie à trois niveaux :

1. Les affiliations à des organisations internationales, tels l'Organisation mondiale du tourisme et le Bureau international du tourisme social BITS.

En effet, à l'OMT, le CIFORT représente l'UQAM (membre affilié depuis 1991) en participant aux assemblées générales de l'Organisation et en étant membre de son Conseil d'éducation. Le CIFORT est reconnu membre officiel au Conseil depuis 2001 depuis l'obtention de l'accréditation TEDQUAL ${ }^{1}$ pour les programmes en gestion du tourisme de l'UQAM, émise conjointement par l'OMT et sa fondation THÉMIS ${ }^{2}$.

Le CIFORT représente aussi l'UQAM auprès du Bureau international du tourisme social par sa participation aux assemblées générales et aux réunions du Bureau.

\section{Le partenariat institutionnel.}

Le milieu international dans lequel nous intervenons amène le CIFORT à s'associer à différents partenaires, soit pour la réalisation de mandats, soit pour des échanges dans le cadre d'ententes bilatérales et plurinationales. Le CIFORT a signé des ententes avec des institutions situées en Amérique du Nord, en Amérique centrale et en Amérique du Sud, en Afrique francophone, en Europe et en Asie.

Par ailleurs, le CIFORT a reçu, pour la réalisation de certains programmes, l'appui financier de divers partenaires, notamment :

- l'Organisation mondiale du tourisme (OMT),

- l'Organisation internationale de la francophonie (OIF),

- le Bureau international du tourisme social - Section des Amériques (BITS-Amériques),

- le Fonds francophone des inforoutes (FFI),

- l'Agence canadienne de développement international (ACDI),

- le ministère des Relations internationales du Gouvernement du Québec,

- le ministère de l'Éducation du Québec (MEQ),

- le Fonds de l'autoroute de l'information du Gouvernement du Québec (FAI).

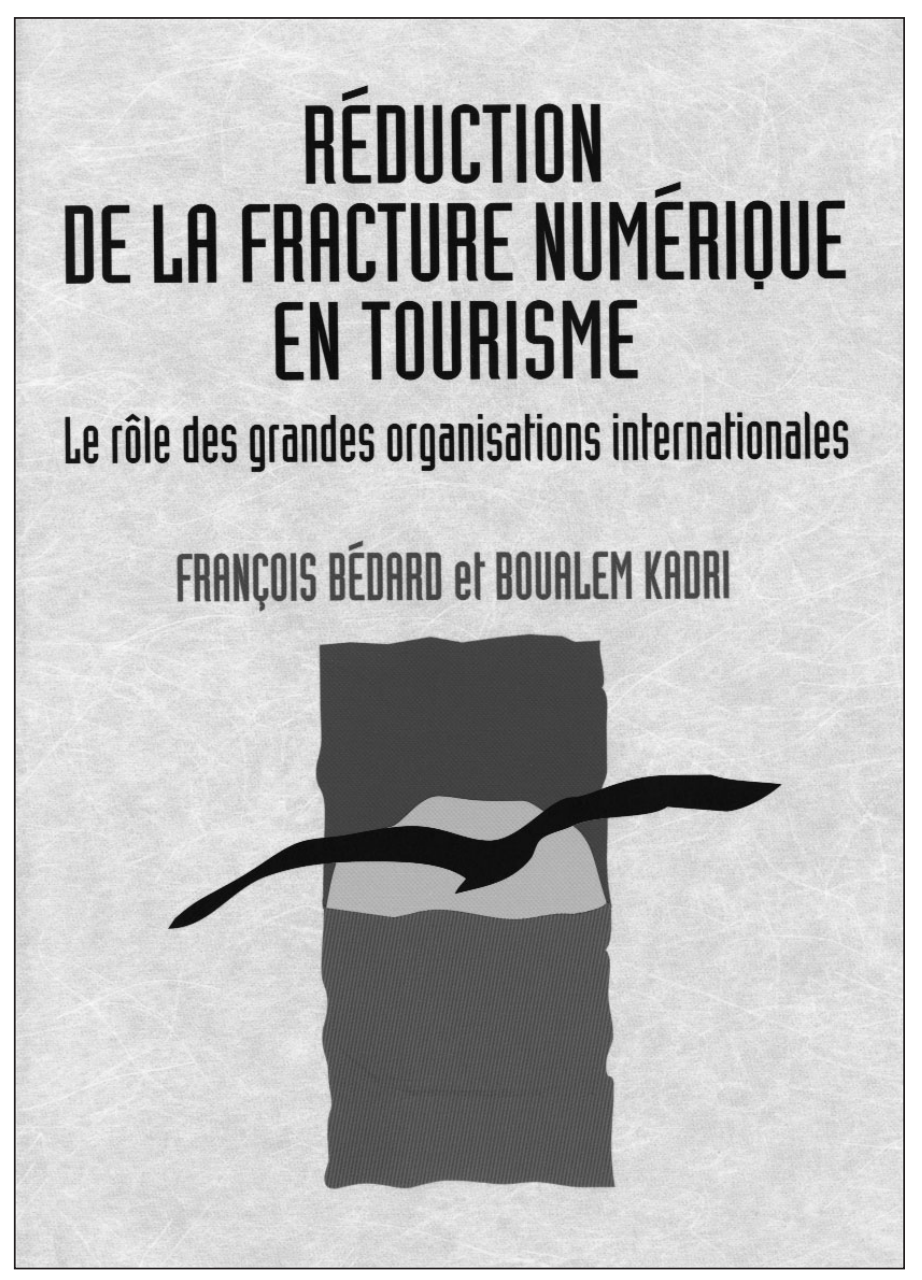

3. La réalisation des programmes à l'international.

En un peu plus d'une décennie, le CIFORT a réalisé un certain nombre de projets à l'international en matière de formation, de recherche, de publication, d'organisation de séminaires et d'accueil de stagiaires. Voici quelques exemples de réalisations complétées et de projets en cours :

\section{Volet formation}

- Honduras : accroissement de la capacité des universités honduriennes à appuyer le développement des communautés locales défavorisées dans une perspective de développement durable (2003-2008).

- Maroc : formation de formateurs en tourisme à l'Institut de tourisme de Tanger (1996), développement d'un observatoire en tourisme à l'Institut de technologie hôtelière et touristique de Marrakech (2003).

- Viêt-Nam : révision du programme de gestion du tourisme (niveau baccalauréat) de l'Université de Dalat (2002).

- Diffusion du cours de l'OMT « Politiques et stratégies en tourisme », sur Internet et cédérom (depuis 2001).

- Rédaction du manuel intitulé «Formation des formateurs en tourisme », en collaboration avec l'Institut de Recherche et d'Études 
Supérieures du Tourisme (IREST) de l'Université de Paris 1 Panthéon Sorbonne (OMT, 1995).

\section{Volet recherche et publication}

- Réalisation d'un numéro de « Téoros international » avec la collaboration des Centres d'éducation et de formation en tourisme de l'OMT (1993).

- Recherche intitulée «Politique de développement du tourisme alternatif en Amérique centrale » en collaboration avec le BITS-Amériques (AUPRICA ${ }^{3}$ : Costa Rica, El Salvador, Honduras. Nicaragua, 1998-1999).

- Recherche sur le tourisme des jeunes aux États-Unis et au Canada, mandat confié par le BITS-Amériques (2000-2001).

- Publication de l'ouvrage Réduction de la fracture numérique en tourisme - Le rôle des grandes organisations internationales aux Presses de l'Université du Québec (François Bédard et Boualem Kadri, août 2003).

\section{Volet séminaires internationaux en tourisme}

- Séminaire «Écotourisme et biodiversité » (Costa Rica 1997).

- Appui à l'organisation du Congrès biennal du Bureau international du tourisme social (Montréal 1996).

- Formation de cadres dirigeants en tourisme de onze pays de la francophonie sur le thème « Tourisme et développement durable ». Collaboration avec l'Institut de Recherche et d'Études Supérieures du Tourisme (IREST) de l'Université de Paris 1 Panthéon Sorbonne (Montréal 1995 et Paris 1993).

\section{Volet accueil de stagiaires (formateurs et étudiants)}

- Accueil de formateurs en tourisme dans le cadre de stages à l'UQAM (Viêt-Nam 1999, 2002 ; Maroc 1994).

- Stages d'études en entreprise pour des étudiants en tourisme notamment de l'Université d'Angers (France), de l'Université de Paris 1 - Panthéon Sorbonne (France), de l'Université Toulouse Le Mirail (France), de La Universidad Autónoma de Guadalajara (Mexique) (1992...).

\section{Quelques exemples de projets des membres du CIFORT pour la période 2003-2005}

- Poursuite du projet sur le développement local et le tourisme au Honduras.

- Projet de recherche-action au Mexique (Cholula) ayant pour objectifs la mise en valeur du patrimoine culturel et religieux de Cholula et l'élaboration d'un plan de développement des infrastructures d'accueil (2004).

- Diffusion d'un programme de maîtrise en administration des affaires (MBA pour cadres) spécialisé « en gestion du tourisme international et des services d'accueil ». Après le premier cycle en tourisme, ce deuxième programme international contribuera à maintenir le leadership de l'UQAM à l'international (2004).
- Développement d'un réseau de veille en tourisme (Maroc et pays méditerranéens).

- Réalisation d'un colloque international sur les systèmes de gestion des destinations (janvier 2004), à Sousse (Tunisie), en partenariat avec l'Institut supérieur des études technologiques de Sousse.

- Projet de réalisation d'un numéro spécial de Téoros sur le Maroc (2005).

Fortement impliqué à l'international par ses diverses réalisations en matière de formation et de recherche, et renforcé par sa reconnaissance institutionnelle formelle comme centre international, le CIFORT contribue de façon significative à accroître le rayonnement international de l'UQAM dans le secteur du tourisme, l'un des secteurs socioéconomiques les plus stratégiques et dynamiques dans le monde en ce début du $\mathrm{XXI}^{\mathrm{e}}$ siècle.

François Bédard est directeur du CIFORT et professeur au département d'études urbaines et touristiques de l'université $d u$ Québec à Montréal. Boualem Kadri , M. Sc., est adjoint de recherche au CIFORT, chargé de cours et doctorant en études urbaines au même département.

\section{Notes}

1 La Certification TedQual de l'Organisation mondiale du tourisme (OMT) est un système unique d'assurance de la qualité offert aux institutions, aux universités, aux écoles de gestion et aux centres de formation reconnus internationalement (site Internet OMT-THEMIS : [www.themis.ad ]).

2 La fondation OMT-THEMIS a pour mission de promouvoir la qualité et l'efficience dans l'éducation et la formation en tourisme et, en général, dans le développement des ressources humaines en tourisme.

3 La Associacion de Universidades Privadas de Centro America. 